\title{
Accurate representation of microscopic scatterers in realistic simulation of OCT image formation
}

\author{
Paweł Ossowski*, Andrea Curatolo ${ }^{\dagger \ddagger}$, David D. Sampson ${ }^{\S \ddagger}$ and Peter R.T. Munro $9 \ddagger$ \\ * Institute of Physics, Nicolaus Copernicus University, 87-100 Torun, Poland \\ $\dagger$ Instituto de Optica "Daza de Valdés", Consejo Superior de Investigaciones Cientficas (IO, CSIC), Madrid, Spain \\ $\ddagger$ School of Electrical, Electronic and Computer Engineering, The University of Western Australia Perth, WA 6009, Australia \\ $\S$ University of Surrey, Guildford GU2 7XH, United Kingdom \\ I Department of Medical Physics and Biomedical Engineering, University College London, WC1E 6BT, United Kingdom
}

\begin{abstract}
There is currently much interest in developing OCT image formation models based on Maxwell's equations. However, all such models face a common challenge: how can microscopic scatterers be represented without limiting the overall size of the sample that can be modelled? Here we show how microscopic scatterers can be accurately represented on computational grids with cells too large to faithfully represent such scatterers using conventional approaches. We also validate this approach experimentally. This approach to OCT image simulation allows for image formation for biological tissues to be simulated with unprecedented realism.
\end{abstract}

\section{INTRODUCTION}

OCT image formation models based on Maxwell's equations are now beginning to be employed to study image formation in optical coherence tomography (OCT) [1]-[4]. The pseudo-spectral time-domain (PSTD) has been has been adapted for this purpose [5] and allows the sample to be discretised using grid cells approaching $\lambda / 2$ where $\lambda$ is the smallest wavelength in the source spectrum. This allows for practically relevant sample volumes to be modelled at the cost of accuracy in representing small scatterers. This limitation was exposed in a recent in silico study of a technique demonstrated experimentally for distinguishing between biological micro-objects using OCT [1]. The in silico study required $\mathrm{TiO}_{2}$ scatterers of diameter $350 \mathrm{~nm}$ to be represented by cubic grid cells with a side of $131.7 \mathrm{~nm}$. The problem of representing such scatterers on a cubic grid is demonstrated in Fig. 1 which shows two possible, näive, discrete approximations to the sphere to be represented. In these cases the cells used to approximate the sphere would be assigned the same refractive index of the sphere $(n=2.52)$. Simulations however showed that these näive discrete approximations result in a very poor representation of the scattering properties of the sphere. To overcome this, an approach was taken where a scatterer was designed, as also shown in Fig. 1, which matched the scattering cross-section of the sphere at the center wavelength of 790nm. Comparison with experiment, however, showed that this optimised discrete scatterer still did not adequately represent the sample being modelled in terms of scattering coefficient and speckle size [1]. In particular, investigation showed that the scattering crosssection of the optimised scatterer differed significantly from that of the the sphere away from the central wavelength, and that the angular scattering properties of the optimised scatterer differed from that of the sphere throughout the entire spectrum.

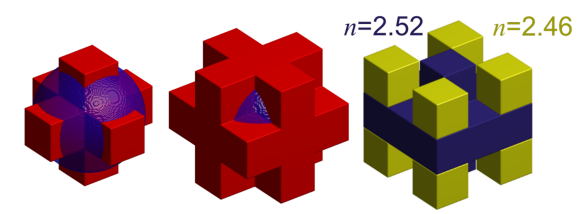

Fig. 1. Two possible näive discrete approximations of a sphere of diameter $350 \mathrm{~nm}$ (left two) and a more complex scatterer designed to match the scattering cross-section of the sphere. In all three cases the cubic grid cells have a side of $131.7 \mathrm{~nm}$.

\section{APPROACH}

\section{A. Simulation noise model}

We assume that shot noise is the dominant source of noise. We describe the optical power guided by the detector optical fiber by $i(k)=\left|\alpha_{\text {sam }}(k)+\alpha_{\text {ref }}(k)\right|^{2}$ where $\alpha_{\text {sam }}(k)$ and $\alpha_{\text {ref }}(k)$ are the mode coupling coefficients from the sample and reference arms, respectively, as a function of wavenumber $k$. The number of photons detected within a spectral band centered on wavenumber $k$ can then be written as $n(k)=\eta i(k)$, where $\eta$ is an effective quantum efficiency. Noise is thus added by replacing each value, $n(k)$, with a value, $\tilde{n}(k)$, which is acquired using a random number generator which takes values from a Poisson distribution with mean $n(k)$. The value of $\eta$ is chosen in such a way that the signal-to-noise ratio, for a region of interest in both the simulated and experimental images, agree. The OCT A-scan can then be reconstructed from the spectrally resolved measurements according to $I\left(z-z_{\text {ref }}\right)=$ $\int_{0}^{\infty} S(k) \tilde{n}(k) \exp \left(i k 2\left(z-z_{\text {ref }}\right)\right) \mathrm{d}(1 / \lambda)$, where $S(k)$ is the effective system spectrum.

\section{B. Structured phantom}

We employed a previously reported phantom [6] designed such that B-scan images of the phantom reveal the letters "OBEL", the " $\mathrm{B}$ " of which is depicted in Fig. 3. Each letter is represented by dispersing $\mathrm{TiO}_{2}$ particles ( $1 \mu \mathrm{m}$ diameter, $n=2.609$ at $\left.\lambda_{0}=1300 \mathrm{~nm}\right)$ at a concentration of $4.5 \times 10^{-3} \mu^{-3}$ into a two-part polydimethylsiloxane 
(PDMS) silicone $(n=1.42)$ resulting in a scattering coefficient of $11.1 \mathrm{~mm}^{-1}$.

\section{Design of discrete scatterers}

The $\mathrm{TiO}_{2}$ scatterers needed to be represented on a PSTD grid with cells of side $1.300 \mu \mathrm{m} / 6=217 \mathrm{~nm}$. A PSTD grid was created and cells either contained within, or intersected by, a cube of side $1 \mu \mathrm{m}$ were allowed to take on a refractive index value of between 1 and 3.5, i.e., different to the background refractive index of 1.42 . The scatterer was assumed to be symmetric in the $x, y$ and $z$ directions which reduced the number of unknown refractive index values to 64 .

The optimisation problem was then setup by surrounding the discretised scatterer with a fictitious cubic surface, $\mathcal{S}_{\text {ref }}$, represented by a collection of triangular facets. The integrated Poynting vector flux propagating through each facet was calculated for the discretised scatterer using the PSTD method and denoted $\hat{S}_{i}(\boldsymbol{n}, k)$, where $i$ denotes the $i$ th facet of $\mathcal{S}_{\text {ref }}, \boldsymbol{n}$ is a 64 element vector of scatterer refractive index values and $k$ is the wavenumber. The same quantity was also calculated using Mie theory for a perfect sphere and denoted $S_{i}(\boldsymbol{n}, k)$. An error functional was then defined as $\epsilon(\boldsymbol{n})=$ $\sum_{j} W_{j} \sqrt{\left(\sum_{i}\left|S_{i}\left(\boldsymbol{n}, k_{j}\right)-\hat{S}_{i}\left(\boldsymbol{n}, k_{j}\right)\right|^{2}\right) / \sum_{i}\left|S_{i}\left(\boldsymbol{n}, k_{j}\right)\right|^{2}}$ where the summation on $i$ is over all facets representing $\mathcal{S}_{\text {ref }}$ and the summation on $j$ is over a sample of wavenumbers from within the spectrum. Each term $W_{j}$ is used to weight the error associated with each wavenumber in proportion with the source power spectral density at wavenumber $k_{j}$. The design refractive index values are then found by computing the value of $\boldsymbol{n}$ which minimises the error functional. In practice this minimisation was performed using Matlab's fminsearch function and the resulting optimised scatterer is shown in Fig. 2 .
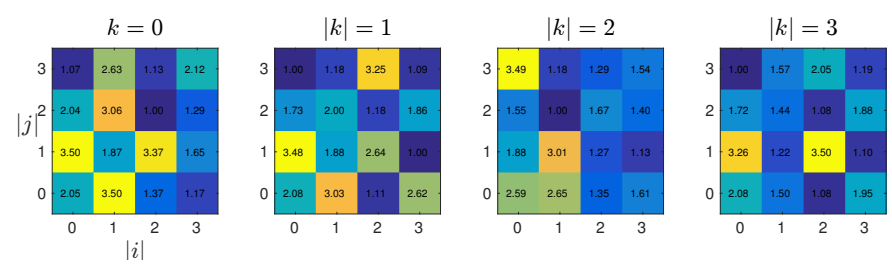

Fig. 2. Rendering of the discretised scatterer refractive index design in planes $z=\Delta k$ where $\Delta$ is the PSTD grid cell dimension.

Simulated and experimental B-scans are compared in Fig. 3 (upper) where the signal-to-noise ratio of the simulated image was chosen to match that of the experimental image. Aside from a slight difference in the design shape of the letter "B", the main difference arising between the two cases is the higher attenuation of the OCT signal evident near the bottom the "B" in the simulated case. The speckle size was analysed using the autocorrelation of the top horizontal part of the "B". The axial and lateral autocorrelation functions for the experimental and simulated cases are plotted in Fig. 3 (lower).
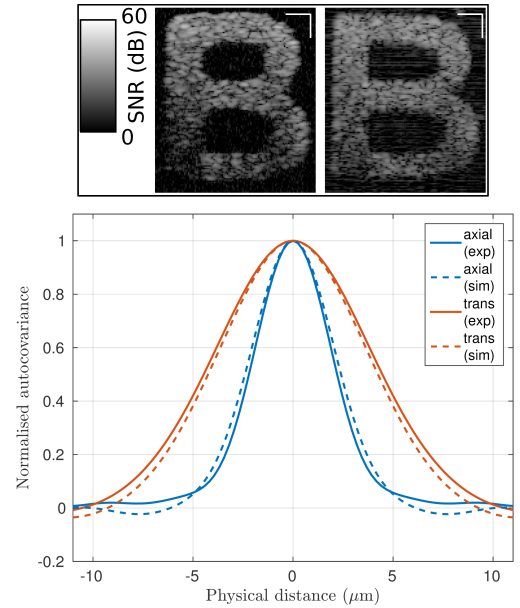

Fig. 3. Simulated (top left) experimental (top right) images of the "B" section of the OCT structured phantom and plots of experimental and simulated autocovariance (lower)

\section{DISCUSSION AND CONCLUSION}

We have presented a method by which scatterers with a dimension approaching the length scale of the computational grid can be more accurately represented in full-wave models of OCT image formation. This is an important development in breaking link between the total sample volume and the finest scatterer detail that can be modelled, a ratio which is generally fixed. The simulated and experimental B-scans shown in Fig. 3 differ principally due to the increased attenuation in the simulated case. It is believed that this stems from uncertainty regarding the refractive index of the $\mathrm{TiO}_{2}$ scatterers. Further simulations should reveal the actual refractive index of the spheres since the scattering cross-section of such spheres varies rapidly as a function of refractive index. Aside from the discrepancy in attenuation ,we obtained good agreement between simulated and experimental speckle size as shown by an analysis of the autocorrelation of the B-scans. It is anticipated that this approach will be used in the future to perform similar in silico studies of practical importance such as that recently demonstrated [1].

\section{REFERENCES}

[1] P. Ossowski, M. Wojtkowski, and P. R. T. Munro, "Classification of biological micro-objects using optical coherence tomography: in silico study," Biomed. Opt. Express, vol. 8, pp. 3606-3626, 2017.

[2] P. R. T. Munro, "Three-dimensional full wave model of image formation in optical coherence tomography," Opt. Express, vol. 24, pp. 27 016-27 031, 2016

[3] P. R. T. Munro, A. Curatolo, and D. D. Sampson, "Full wave model of image formation in optical coherence tomography applicable to general samples," Opt. Express, vol. 23, pp. 2541-2556, 2015.

[4] T. Brenner, D. Reitzle, and A. Kienle, "Optical coherence tomography images simulated with an analytical solution of maxwell's equations for cylinder scattering." J. Biomed. Opt., vol. 21, 45001, 2016.

[5] P. Munro, D. Engelke, and D. D. Sampson, "A compact source condition for modelling focused fields using the pseudospectral time-domain method," Opt. Express, vol. 22, p. 5599-5613, 2014.

[6] A. Curatolo, B. F. Kennedy, and D. D. Sampson, "Structured threedimensional optical phantom for optical coherence tomography," Opt. Express, vol. 19, pp. 19480-19485, 2011. 